\title{
Carnets
}

Revue électronique d'études françaises de l'APEF

Deuxième série - 7| 2016

Plurilinguisme et migrations dans la littérature de langue française

\section{Le Dédale des Disciples de Rémy Tissier}

Entre « Hétérarchie » linguistique et transgressivité littéraire

\section{Mamadou Faye}

\section{(2) OpenEdition}

\section{Journals}

Édition électronique

URL : http://journals.openedition.org/carnets/1011

DOI : $10.4000 /$ carnets. 1011

ISSN : 1646-7698

Éditeur

APEF

Référence électronique

Mamadou Faye, "Le Dédale des Disciples de Rémy Tissier », Carnets [En ligne], Deuxième série -

7 | 2016, mis en ligne le 31 mai 2016, consulté le 30 avril 2019. URL : http://journals.openedition.org/ carnets/1011 ; DOI : 10.4000/carnets.1011

Ce document a été généré automatiquement le 30 avril 2019.

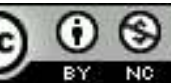

Carnets est mis à disposition selon les termes de la licence Creative Commons - Atribution - Pas d'utilisation commerciale 4.0 International. 


\title{
Le Dédale des Disciples de Rémy Tissier
}

\author{
Entre « Hétérarchie » linguistique et transgressivité littéraire
}

\section{Mamadou Faye}

1 Nous voudrions, à travers cet article, examiner, dans une perspective d'hétérogénéité et de transgressivité appliquée au plurilinguisme, le livre de Rémy Tissier, Le Dédale des disciples (2004). Mais auparavant, afin d'apporter une réponse assez fourbie à cette question, il nous paraît méthodologiquement approprié de procéder succinctement à quelques remarques préliminaires. En guise de décryptage du mot-valise « hétérarchie ", il convient d'ores et déjà de relever que celui-ci est formé des substantifs " hétérogénéité » et « hiérarchie ». Ce concept inédit, presque un hapax dans le champ de la critique, nous est inspiré de Douglas Hofstadter (1985) qui l'a forgé pour désigner une « hiérarchie désacralisée d'où toute idée de priorité s'est évanouie » (Westphal, 2007 : 14), d'où toute notion d'infrangibilité est ruinée et abolie pour l'émergence d'une égalité des hiérarchies, pour leur nivellement intégral.

2 En faisant fi du primat des structures hiérarchiques au plan linguistique, des écrivains en sont arrivés à dénier au concept de hiérarchie son autorité, et à travailler à son détrônement. Sous l'effet de leurs faits d'artiste, de créateurs, ce concept voit son autorité déboulonnée, son aura délitée et désagrégée : ou bien la verticalité devient étale, ou bien l'horizontalité devient verticale. Déchue, la «transcendance» intègre les rangs, la hauteur en prend pour son grade, et l'horizontalité interactive s'exhausse au même niveau que la verticalité canonique. L'opération de déstabilisation des grilles séparant les hiérarchies peut correspondre à la transgressivité, à la qualité de ce qui est transgressif, à ce qui a la faculté de poser des actes transgressifs, qui peut opérer des transgressions.

Appréhendée sous l'angle de la géologie, la transgression se rapporte au mouvement de la mer qui déborde sur les aires continentales avoisinantes. Mais dans l'usage courant, la transgression fait habituellement référence à la violation d'un interdit, au fait de passer outre une loi, ou encore à un refus d'obéissance. Adeptes des chemins de traverse, les créateurs aiment prendre des risques. Pour eux, la transgression est libératoire et permet de s'affranchir des limites du conformisme (Ventura et Mottet, 2009 : 7). 
En nous appuyant sur la linguistique moderne, la sémiotique, l'anthropologie, la psychanalyse et la critique d'art, entre autres, nous nous pencherons sur le refus d'imposition ou de soumission qu'implique « l'hétérarchie » sur le strict plan linguistique. Suivant le second versant de notre analyse, nous verrons que l'écrivain, rétif aux hiérarchies, caresse le dessein et le destin d'aller au-delà des semblances fixées pour semer le bon grain des vertus préventives des conflits de culture.

\section{« Hétérarchie » linguistique}

4 Durant les décennies passées, le roman s'est distingué par ses crises - de croissance peutêtre - répétées. De telles crises affectent ses éléments constitutifs : le personnage, la narration, l'illusion romanesque, la langue. Nous voudrions nous attacher à deux aspects et à un moment circonscrit de cette pandémie atteignant la presque totalité de la sphère romanesque : la crise linguistique et celle affectant la narration. Le pied de nez fait à la norme unitaire et fédératrice, « les lignes de fuite, les mouvements de déterritorialisation et de déstratification » (Deleuze et Guattari, 2013:10) constatés par Deleuze, sont, entre autres, les symptômes d'une telle crise. Ce sont ces dérèglements et ralliements notés aux niveaux linguistique et narratif qu'il importe d'étudier ici. Il convient de préciser que le plurilinguisme est à entendre comme un aménagement de deux ou plusieurs langues et constitue un puissant, voire prodigieux, facteur d'ouverture. Dans le contexte de "l'hétérarchie », le plurilinguisme relèverait d'« une organisation acentrée d'une société de mots » (Rosenstiehl et Petitot, 1974 :27) et, pour paraphraser Alain Finkielkraut, il n'est pas à considérer comme attributif mais comme conjonctif, c'est-à-dire qu'il s'exprime dans le « et », non plus dans le « est » (Finkielkrault, 2000 : 76). Plus simplement, il s'agit de hiérarchies qui se neutralisent les unes les autres à travers la diversité née de leur croisement fertile.

5 Mais «l' hétérarchie » n'a d'intérêt qu'en tant qu'elle désigne une mise en commun, un système associatif. Par conséquent, l'objet du présent article n'est pas de se limiter à constater la valorisation des hétérogénéités par la dissolution ou le nivellement des hiérarchies, ou leur résorption dans une catégorie supérieure comme celle des langues, mais bien d'étudier leur mise en jeu, leurs manipulations et leurs frictions, en vue d'explorer leur fertilisation croisée. La notion est doublement paradoxale car, à considérer le seul mot hétérogène, on fait tout de suite un énoncé paradoxal si, effectivement, on s'appuie sur la définition qu'en propose l'étymologie : d'après celle-ci, est hétérogène ce qui est constitué de genres différents. En nous appuyant sur la loi du genre que Derrida définit ainsi : «dès que du genre s'annonce, il faut respecter une norme, il ne faut pas franchir une ligne limitrophe, il ne faut pas risquer l'impureté, l'anomalie, la monstruosité » (Derrida, 2003 : 35), nous prenons mieux toute la mesure de cette contradiction. Et comme tout énoncé paradoxal, "l'hétérarchie» a une valeur heuristique propre à étudier le mouvement de débordement des limites, quand les hiérarchies se lézardent ou craquent aux coutures. Un tel réseau de craquelures se traduit par les solutions de continuité qui sont notées dans le récit et qui sont symptomatiques de « l'hétérarchie ». Ces ruptures fonctionnent comme des alternances codiques, c'est-à-dire comme «la juxtaposition à l'intérieur d'un même échange verbal, de passages où le discours appartient à deux systèmes ou sous-systèmes grammaticaux différents " (Thiam, 1997). Ce franchissement, qui fait écran et nuit à l'unité et à la cohérence linguistique, s'observe principalement à deux niveaux : 


\section{Au niveau des langues maternelles ou locales}

6 Les mots des langues maternelles renvoient, selon la terminologie de Gobard, au « langage vernaculaire» (Gobard, 1976) puisque parlé spontanément, et moins fait pour communiquer que pour communier (nous avons, par exemple, le mot pulaar Djamgwaeli, 2004 : 110). Quant aux mots arabes, ils tiennent du langage «mythique» (Gobard, 1976) et/ou religieux (l'alif, lam, xalima, le calam, 2004 : 110). Le premier niveau se complète par les termes wolofs dont le récit est jonché. Forte de sa densité consécutive au fait qu'elle est parlée à l'échelle de la ville de Ndar (autre nom de Saint-Louis du Sénégal) et même nationale, cette langue wolof acquiert le statut de langage « véhiculaire ». Par l'essaimage de ses mots dans le récit, cette langue semble rivaliser âprement avec la langue française. Sa forte présence dans le récit serait emblématique de son aura dans la société sénégalaise et saint-louisienne particulièrement. C'est pourquoi Tissier y voit un langage ancillaire : « le wolof est la langue ancillaire de lîle » (2004: 63). Tout comme le langage "véhiculaire» de Gobard, celui qualifié d'«ancillaire» par Tissier est destiné à la communication dans les centres urbains.

7 Mais les opérations de «l'hétérarchie » s'illustrent surtout à travers la juxtaposition de l'énoncé du mot ou du syntagme wolof et de sa transcription immédiatement suivie de sa traduction, ou inversement : « Nio ko bok, je vous en prie » (2004: 87); « On dit debout et couché, taxaw ak tëdd» (2004: 122). Ce faisant, Tissier sacrifie au xénisme propre à l'hétérolinguisme et qui a pour fonction d'indiquer, contrairement au pérégrinisme ${ }^{1}$, la présence d'un élément étranger dans une langue quelconque, généralement suivi d'une explication métalinguistique. Ainsi, le xénisme sera plutôt "une insertion d'origine étrangère souvent accompagnée - sinon toujours - d'une glose qui annule l'impression de faute » (Lawson-Hellu, 2003 : 318). Toutes ces deux opérations sont délibérément inscrites sur le même pallier et mises sur le même pied. Cela, loin d'être fortuit, figurerait une volonté de construire des hiérarchies convergentes, une hétérarchie. Cette démarche pédagogique qui viserait à enseigner les langues locales va jusqu'à toucher la didactique de la phonétique corrective. C'est ainsi qu'appréciant l'instrument à vent utilisé chez les Bassari, la femme du personnage principal trahit sa difficulté à articuler convenablement le nom de l'objet. Son compagnon s'en est offusqué, qui n'a pas manqué, non sans zèle, de sonner l'alerte : « J'ai essayé, pour que nous puissions garder la mémoire de cette flûte... Ce n'est pas une flûte! C'est un Ektümbó, prononce Lorenzo, comme expectorant la dernière syllabe, le "bó " " (2004: 148). Du coup, la fiction mord sur la diction et renverse le rapport qui était de mise entre langue des dominants et langue des dominés: désormais chacun apprend la langue de l'autre, et vice-versa. La balance est ainsi maintenue égale - du moins à titre principiel - entre l'intérêt à comprendre la langue locale et celle étrangère.

8 L'avantage de cet équilibre est de trois ordres: d'abord servir de bouclier aux langues locales dans un contexte où celles-ci sont menacées de disparition, happées qu'elles sont par une mondialisation assurant exclusivement la promotion des langues dites internationales composées respectivement des langues considérées comme hypercentrales (l'anglais, en l'occurrence) et celles qualifiées de centrales (l'espagnol, le portugais, entre autres). Cela risque de faire perdre aux langues locales leur âme ou même d'entraîner le «matricide [qu'il y a] dans l'abandon d'une langue natale » (Kristeva, 2007: 7). "L'hétérarchie » mène ainsi le combat contre une certaine 
"glottophagie" rampante, qui s'épanouit insidieusement tel un fruit vénéneux, si l'on retient, selon une révélation de l'Organisation Internationale de la Francophonie, que »sur les presque 7000 langues que nous connaissons aujourd'hui, la moitié est appelée à disparaître au cours de ce siècle » (La Langue française dans le monde, 2010 : 267). Certaines langues, d'ailleurs, ont déjà fini ensablées comme un petit oued. Ensuite, il permet de s'enrichir - immatériellement -: la connaissance de plusieurs langues contribue à l'épanouissement non seulement moral et affectif, mais aussi intellectuel de ceux qui les parlent. Enfin, il conduit à s'émanciper de la première langue et à se prémunir contre le risque de fermeture d'esprit, de méfiance vis-à-vis de l'étranger, d'autosatisfaction, et d'absolutisme qui menace tout unilingue. Parler diverses langues renouvelle l'art de communiquer et de vivre comme il revigore la créativité. Ainsi que le perçoit Van Vlasselaer,

une culture autre est une culture différenciée du monde (...). Une lecture

"dédoublée" de la réalité ne peut que déployer celle-ci, l'ouvrir (...). Alors [la]

disponibilité à saisir le différentiel permettra une célérité, une vélocité qui (...)

ouvriront à la créativité (Van Vlasselaer, 1994 : 109).

Mieux, l'arrivée (non l'intrusion) d'une nouvelle langue assure une fonction d'élévation: elle hisse d'un cran la langue première, il la tire hors de la sphère ténébreuse de l'absolutisme en la relativisant. L'enrichissement subséquent rehausse l'identité en la rendant plus consciente d'elle-même, en la remodelant.

C'est peut-être dans cette optique que s'inscrit l'intérêt à vivifier la langue, à lui donner un destin supérieur, à la transmuer en une chose ailée, en lui prêtant «l'orgueil d'accomplir ce que fut d'abord le projet idéal des abeilles natales. Voler plus haut que les parents: plus haut, plus vite, plus fort» (Kristeva, 2007: 7). L'image ornithologique employée par Kristeva suggérant que la langue n'a cure des frontières, est aussi celle qu'utilise Tissier qui insère dans le récit cette réflexion par l'entremise de son personnage : « une langue n'est-elle pas comme un oiseau ? Elle a besoin de pattes pour marcher sur son propre terrain, sinon son terroir, mais elle a aussi, surtout, besoin d'ailes pour être lue le plus loin possible ...» (2004: 79). Ainsi, la langue est une réalité hétérogène, dynamique et horizontale, en perpétuel déplacement vers l'inconnu. L'image de l'ornithologie ${ }^{2}$ commune à Kristeva et à Tissier renverrait à ce que Lotman appelle la » zone du dynamisme sémiotique [qui rend] la frontière (...) bilingue et polyglotte » (Lotman, 1999)3. Au sein d'un tel espace, plus le signe s'éloigne du centre de la sémiosphère, plus il perd de sa fixité et plus il devient flottant. La force centrifuge à laquelle est soumise cette sémiosphère présente des zones d'indéterminations, et donc des hétérogénéités. Le plurilinguisme chercherait ainsi à abolir la relation hiérarchique entre les langues afin de créer les conditions amènes d'un vivre-ensemble, en vue de faire prospérer un colinguisme ouvert, inclusif et dynamique. Lequel colinguisme permettrait aussi de prévenir et d'endiguer l'érosion de situations conflictuelles, mais surtout de capter le potentiel évolutif du métissage des cultures.

11 Face à un tel enjeu, le roman se fait volontiers processionnel en faisant escorte à ce processus par lequel chacun, en s'extirpant du cocon douillet de sa propre langue, de sa sphère sémantique individuelle, consent à cheminer vers un univers collectif, pour apporter ainsi sa petite pierre à la fusion de diverses constructions du monde en un modèle de référence commun. L'auteur engage ainsi une médiation, une négociation par son texte pour « une mise en contact de [plusieurs] langues-cultures » (Syn-thèse, 2010 : 7). Enfin, les langues locales contribuent à faire acquérir à Tissier et à tout autre étranger 
virtuel, non pas une identité unique, stable et statique, mais une identité plurielle, contrapuntique, qu'Edgar Morin désigne par la formule "identités concentriques » (Morin, 2002). Français natif, Tissier parle forcément anglais puisqu'il vit aux Etats-Unis, et, à la faveur de son séjour en Afrique, le voilà en plein apprentissage de certaines langues africaines et même de l'arabe. L'on ne saurait tout de même passer sous silence la non maîtrise, par Tissier, de l'orthographe des langues africaines. En font foi les irrégularités relevées dans la transcription de certains mots wolof (jërëjëf au lieu de " jerejef», 2004 : 176 ; njiitu xaleyi et non " njït u xaléyi », 2004 : 155, etc.). Si de telles erreurs ne sont pas volontaires, et qu'elles n'ont pas pour effet de marquer un certain niveau d'apprentissage de l'auteur, alors les éditions L'Harmattan pourraient être invitées à une plus grande vigilance.

\section{Au niveau des constructions lexicales}

Celles-ci portent sur le français « langue référentiaire », suivant toujours la terminologie de Gobard. En plus des alternances codiques dont une des fonctions est de mettre sans cesse la pensée en porte-à-faux, on relève des barbarismes revendiqués et assumés ( famillicides, baguetterie), des néologismes par lesquels la langue glisse ici dans les coulisses d'une logique récupératrice. Quant aux régionalismes (le mot gadjé par exemple), ils rendent le français dynamique et ouvert à la créativité en l'inféodant à une logique refondatrice et actualisante. Ils empêchent ainsi que la langue française souffre d'un marasme lexical, d'un manque criard de vocabulaire pour transcrire dans le langage quotidien les réalités nouvelles ou anciennes du monde. Ces néologismes comptent au nombre des initiatives novatrices visant à pallier ce que Marc Chevrier appelle « la fatigue linguistique de la France » (Chevrier, 2012). Pour se remettre de cette langueur dont elle souffre, pour s'offrir un ballon d'oxygène, la langue française a tout intérêt à se faire poreuse à l'instar du langage wolof, dont le narrateur dit qu'il est « absorbant comme une éponge sèche, émaillé de mots étranges et étrangers » $(2004: 16)$.

Cette étrangeté, pouvant aller jusqu'à relever d'une "langue "fôtive" de l'hybridité linguistique » (Cardonne-Arlyck, $2001: 313)$, témoigne d'une intentionnalité à déstabiliser le système à partir de l'intérieur. Puisque la vie elle-même n'est guère juste, il n'y aurait apparemment pas de raison que les mots le soient. Pareille déstabilisation serait consécutive à une "recherche d'une dynamique du déséquilibre indicatrice d'un type supérieur d'évolution systémique " (Prigogine et Stengers, $1984: 166)$ et/ou linguistique propre à mimer la diversité et l'incohérence des choses. L'auteur, convaincu sans doute qu'aucune langue vivante ne saurait se développer en circuit clos, a dû adopter " une méthode de type rhizome [qui] ne peut analyser le langage qu'en le décentrant sur d'autres dimensions et d'autres registres" (Deleuze et Guattari, 2013: 14). Face à l'incapacité (réelle ou feinte) à dire les choses conformément à la norme linguistique, l'auteur a recours au pouvoir créatif du langage dans la résolution de problèmes, à ses principes de poéticité et de communication esthétique. Cela s'accomplit au prix d'une perturbation des codes, d'une posture défiante et déviante, d'un dérèglement représentant une sur-écriture, "un surcodage» (Eco, 1975: 155). La créativité ou l'anomie créatrice dont procède la performance est à l'origine de ces barbarismes. Le barbarisme, qui se manifeste à travers la performance linguistique, devient ainsi un catalyseur, une force perturbante permettant d'établir une opposition entre les modèles 
établis, conventionnels et des modèles alternatifs, entre la culture et les éléments refoulés, latents, rejetés dans la non-culture.

Cette dernière se trouve valorisée comme un système protoculturel, c'est-à-dire comme source de culture nouvelle ou de culture renouvelée. Aujourd'hui, c'est la non-culture qui a été à la base de la codification des mots tels que "essencerie ", "gouvernance", "primature », « négritude ", etc.). Cette déstabilisation figure la capacité de la langue à glisser hors des conventions pour entrer dans une zone de régénérescence où ce qui eût été étouffé et refoulé deviendra digne des «mots de la tribu ( (Mallarmé, 1887), et donc sera accepté et intégré dans le poreux circuit linguistique. Elle actualise aussi le processus évolutif décrit dans la sémiotique culturelle de Tartu par laquelle la non-culture peut devenir « un puissant stimulant du développement culturel » (Ivanov, Lotman, Ouspenski, Piatigorski, Toporov, $1974: 125-156)$. C'est dire que l'identité culturelle est en permanente construction, et qu'elle n'est effectivement conquise que lorsqu'elle s'ouvre à ce qui la dépasse. Cette constance et cette persistance dans la capacité à s'illustrer par d'autres mots confèrent à «l'hétérarchie » son pouvoir transgressif et sa dimension prévisionnelle des conflits de culture.

\section{Vertus préventives du parti pris transgressif}

L'intitulé de ce point rappelle, non sans raison, le livre Résoudre des conflits de culture, que nous devons à Roger Parent (2009). La procédure essentielle du Canadien consiste à localiser et à explorer le rapport ou l'entrecroisement entre le versant sémiotique et celui culturel des conflits. Dans le roman tissierien, l'écart noté entre la performance et les conventions confère au récit son originalité, sa transgressivité ainsi que son spectre culturel et sémique : le plurilinguisme, dépassant les revendications linguistiques, arrive aux revendications culturelles et, probablement, cultuelles. Allant bien au-delà de la posture de Parent, Tissier s'arme de la volonté sous-jacente, non de résoudre, mais d'élaborer une stratégie d'anticipation qui requiert que la langue soit une entité vivante voire dynamique. Ainsi, le Français-Américain pourrait partager la conviction deleuzienne selon laquelle l'essence d'une langue est qu'elle garde vive la flamme de la plasticité, qu'elle fait constamment usage de ses ressorts pour un dynamisme toujours plus marqué : " une langue ne se referme jamais sur elle-même que dans une fonction d'impuissance » (Deleuze et Guattari, 2013 : 14). La langue dispose d'un potentiel, d'une réserve qu'on pourrait dire abyssale, elle constituerait un « silo [sans fond] où se préserve et murit» (Césaire, 1983: 46) une infinité de virtualités, un gisement de ressources renouvelables aussi longtemps qu'il existera des locuteurs et des artistes. En plus, l'on a $\mathrm{pu}$ voir que la langue, tel un tubercule, agglomère des performances langagières très diverses, qu'il n'y a pas de langue en soi, verrouillée et définitive, ni d'universalité du langage, mais qu'il existe un concours de langues particulières, de dialectes et de provincialismes.

16 Par voie de conséquence, il n'y a pas de locuteur-auditeur idéal, pas plus de communauté linguistique homogène, sauf l'Adam mythique, le seul qui ait échappé au dialogisme inhérent au langage (Bakhtine, 1978). La formule chère à Weinreich trouve toute sa pertinence ici, lui qui perçoit la langue comme » une réalité essentiellement hétérogène » (Deleuze et Guattari, 2013 : 14). Mieux encore, à cette qualité qui est le sceau, la marque distinctive de la langue, s'ajoute une deuxième spécificité : contrairement à la religion, la langue n'a pas vocation à être exclusive. On peut pratiquer à la fois le portugais, le 
français, l'arabe, l'hébreu, le sérère, le bassari. En revanche, l'on ne saurait être à la fois catholique, luthérien, musulman et juif. Un autre élément de comparaison réside dans le fait qu'un homme peut vivre sans aucune religion, mais jamais sans aucune langue. Adam Pollo du Procès verbal de Le Clézio, s'étant volontairement mis au ban de la société, «fier de ne plus avoir grand-chose d'humain» (1963: 22), vit terriblement seul, sans religion. Mais il use du langage en communiquant oralement, et par voie épistolaire, en français ; parfois même, il écoute des chansons anglaises ${ }^{4}$. Il en est de même des habitants de Kédougou où le héros tissierien, venu comme pour porter et prêcher la bonne parole altéritaire, a rencontré une communauté constituée d'êtres « intègres [qui] ne sont ni musulmans ni chrétiens ", mais qui n'en sont pas moins valeureux, pas moins heureux ni moins dignes (2004: 142).

L'autre enseignement que l'on pourrait tirer de la langue en tant que réalité hétérogène par nature, c'est que, suivant la remarque d'Amin Maalouf, la langue, en raison notamment de sa qualité bifide, jouit de « cette merveilleuse particularité d'être à la fois facteur d'identité et instrument de communication» (Maalouf, 1998: 153). D'où le caractère inextricable du lien entre le linguistique et l'identitaire - un rapport dont l'extrême étroitesse est soulignée à la faveur de la redondance - : la langue a « vocation à demeurer le pivot de l'identité culturelle, et la diversité linguistique le pivot de toute diversité » (Maalouf : 153). Il n'y a certes pas de hiérarchie entre les langues, ni d'ordre absolu des langues différentes; il n'y a pas non plus de différence catégoriale entre ces langues. Cependant, il y a peut-être lieu de convenir que cette absence d'ordre absolu des langues ne doive pas pour autant faire penser qu'elles seraient toutes nées égales. En fait, elles ne sont égales que du point de vue du besoin d'identité.

En effet, placées sous l'angle identitaire, elles remplissent toutes exactement le même rôle comme elles ont toutes également droit au respect de leur dignité. Si les langues méritent d'être reconnues égales en dignité, c'est certainement en raison du fait que chaque culture œuvre pour la création de sens, pour se faire pourvoyeuse d'une signification complémentaire, locale certes, mais nécessaire à l'élaboration du sens global. Sous ce rapport, suivant la précision de Touraine,

[1]e sens construit par chaque culture est différent, mais il ne peut y avoir de communication entre les cultures que si chacune reconnait dans les autres la construction d'une partie du sens de l'expérience humaine. [Par conséquent], le multiculturalisme n'est pas l'adversaire de l'universalisme, (...); il en est l'autre face. Ce qui unit l'universalisme et le multiculturalisme est plus important que ce qui les oppose, car ce qui les unit est la commune volonté de placer une culture audessus des pouvoirs d'un État ou des intérêts d'un groupe social (Touraine, 1997 : 291-319).

Toutefois, si on la circonscrit autour de sa fonction d'instrument d'échange, cette égalité qu'on escompte du point de vue identitaire s'estompe et s'efface au regard du bassin où elle se déploie comme langue de communication. La langue est alors fondamentalement dyadique : elle sépare et unit tout à la fois ; elle est à la fois le maillon et la chaîne, ce qui distingue les hommes et ce qui les relie ${ }^{5}$.

Un autre type de langage qui participe du plurilinguisme et de l'égalité est le langage du corps. Celui-ci est hissé par la narration au statut de langue trait d'union, langue commune par excellence. Voici comment le roman suggère l'existence d'un tel langage, relevant de la pragmatique de la communication humaine, une sorte de synthèse de toutes les langues à laquelle ont recours ces deux personnages appartenant à deux communautés linguistiques différentes, mais habités par une même soif d'empathie : 
La femme de Balingo ne parlait pas français mais, avec elle, j'ai vraiment senti que j'étais bienvenue! Nos visages se sont rapprochés, nos fronts se sont touchés, j'ai pu voir de près les tatouages qu'elle avait autour des yeux. Elle m'a ensuite noué au poignet le bracelet de perles impossible à dénouer que je porte aujourd'hui ! Je me suis vraiment sentie proche d'elle... (2004:151).

21 En rapportant ce propos, Tissier semble s'inscrire aussi dans la lignée de Watzlawick et de Rjudolf Carnap qui ont proposé un modèle selon lequel « les deux termes, communication et comportement [sont] pratiquement synonymes» (Wattzlwick, Beavin, Jackson, 1972 : 16) ; qu'il s'agisse d' « Activité ou [d'] inactivité, [de] parole ou [de] silence, tout a valeur de message" (Ibid.: 46). Comme l'indique l'emploi des verbes pronominaux à valeur de réciprocité («se sont rapprochés»; »se sont touchés»), il existe, en plus de cette conception de la communication en tant que comportement, celle de la communication comme interaction. Le rapport entre l'émetteur et le récepteur constitue une métacommunication qui, de nature analogique ou non verbale, coexiste avec le mode généralement digital ou verbal du message de façon à valoriser ou à neutraliser le contenu de l'énoncé. Ce contenu se trouve alors tributaire de la métacommunication de la relation. Ce témoignage montre la capacité du corps à se faire entendre de tous et de chacun, comme s'il était le point focal de toutes les langues, leur pédoncule. Par ce comportement renvoyant à la proxémique ${ }^{6}$, à la faveur de ce geste kinésique, le romancier, tout en somatisant et spatialisant le langage, semble voir dans le corps et dans l'espace physique un potentiel de communication supplémentaire.

Ce type de langage, pour le moins singulier parce que amputé de sa face acoustique, pourrait résoudre et surmonter la dichotomie qui assimile la langue à un manteau dyadique, c'est-à-dire synonymique de libération et d'obstacle. En effet, l'obstacle que pourrait constituer la langue en tant qu'expression vocale se voit écarté par cette forme d'expression averbale. Ce type de langage relevant d'attitudes «exercées derrière ces portes closes que sont les lèvres » (Bronckart, 1987 : 21), d'autres systèmes signifiants, réduit à néant la frustration de ne pouvoir communiquer par les mots. "Si on parle seulement avec les mots, on n'est pas libre »(Le Clézio, 1973 : 17). En outre, en valorisant seulement la communication linguistique, l'homme nie sa propre corporalité qui fait partie du grand Tout cosmique matériel. Il a surtout le mérite de montrer que l'essentiel de la communication n'est pas le message, mais que c'est plutôt la relation ; il ne s'agit pas seulement de reconnaître le droit à la différence, mais de prendre conscience de l'enrichissement qui résulte de toute relation intersubjective. Cela illustre le concept de " poétique de la relation » développé par Édouard Glissant dans son essai philosophique éponyme et qui met en avant une poétique ouverte, multilingue et en prise avec le tout possible. (Glissant, 1990 : 108-109)

Tissier partagerait le rêve de Le Clézio qui propose une forme de communication totale et éclectique. Dans L'Inconnu sur la terre, ce dernier écrit :

Si le langage n'est fait que de mots, il n'est rien du tout (...). Mais quand dans les mots viennent la danse, le rythme, les mouvements et les pulsations du corps, les odeurs, les traces tactiles (...) quand les mots jaillissent non seulement de la bouche mais du ventre, des jambes, des mains (...) quand surtout les yeux parlent, et le regard est une route sans fin qui traverse le cosmos ; on est alors dans le langage, dans la beauté. (Le Clézio, 1978 : 106)

Le propos de Sarha pourrait aussi figurer la nécessité d'encourager la diversité linguistique par laquelle chacun pourrait parler, en sus de sa langue identitaire, d'autres langues. Telle paraît être la voie royale de la sagesse pour peu qu'on soit épris du souci de 
faire chuter l'enclos identitaire, d'éviter la rétraction identitaire, d'apprivoiser et de tenir en laisse la panthère identitaire. Cette voie paraît constituer la condition unique pour «tirer du formidable essor des communications l'enrichissement, à tous les niveaux, plutôt que l'appauvrissement, plutôt que la méfiance généralisée, et le trouble dans les esprits" (Maalouf, 1998: 164). "Dans une optique de culture de la paix, il faut", recommande Raymond Renard conscient, à l'instar d'autres penseurs, que le flirt avec les communautarismes n'est guère étranger au heurt des cultures, "associer l'altérité et l'identité ; leur alliance est nécessaire. Sous peine de fondamentalisme. » (Renard, 2000 : 49)

\section{Conclusion}

Du sujet qui vient d'être passé au crible de l'analyse, il apparaît que la question du plurilinguisme et de l'hétérolinguisme a fourni à l'imagination de Tissier des dimensions qui satisfont pleinement à sa perception de l'identité et à sa conception de la littérature. Aussi, son roman correspondrait-il à une entreprise de textualisation des langues à la racine de l'acte créatif. Une entreprise qui lui aura permis de mettre en évidence la diversité des faits et effets de langue altéritaire selon la postulation d'une relation égalitaire. L'on aura noté, en particulier, diverses embardées de type linguistique, formel, qui font baver le récit dans les marges, en entrainant des craquements aux coutures et aux contours pour le ralliement d'autres langues.

De telles transgressions mettent en valeur le fait que l'essentiel d'une langue n'est pas dans la fixité, mais dans son dépassement, son déplacement. Tissier revendique une liberté créatrice inscrite dans le processus d'une valorisation de l'anomie créatrice qui, toutefois, se doit d'être encadrée pour éviter qu'elle prenne les chemins de traverse menant vers la tératologie. Pour Tissier, la transgression est libératoire car elle permet de s'émanciper des limites des conventions, de rompre les rets du conformisme, et de congédier les hiérarchies ainsi que les orthodoxies. Pour y arriver, Tissier aura transcendé son statut d'auteur en se faisant ôteur de normes. Mais il s'agit de pratiques poreuses à divers éléments qui, ayant profondément irrigué le récit, ont contribué à revigorer le plurilinguisme pour favoriser un vaillant métissage, une fertilisation croisée entre langues africaines, occidentales voire asiatiques. Elles intègrent des jeux de miroir, des emprunts, des juxtapositions, des métamorphoses et des hybridations. Une telle intégration pourrait figurer une sorte de mystique de la transgression séminale d'un nouvel ordre linguistique mondial où les hiérarchies et les ordres ne vaudront plus que par leur absence, ne croîtront plus que sur le sol de leur propre absence.

\section{BIBLIOGRAPHIE}

BRONCKART, J. P. (1977). Théories du langage / Un introduction critique. Liège : Mardaga.

BAKHTINE, Mikhail (1978). Esthétique et théorie du roman. Paris : Gallimard. 
BENAYADA, K. \& al. Pratiques de la transgression dans la littérature et les arts visuels (2009). Actes du colloque international : Université d'Orléans « Les transgressions verbi-voco-visuelles », [date de consultation : le 11 / 03 / 2015]

$<U R L$ : http://www.imgga.com/search/?q=pratique+de+la+transgression+dans+la+litt\% 25u00e9rature\&cX=partner-pub6879640819915772\%3A1767833448\&cof=FORID\%3A11\&ie=UTF-8.>

CARDONNE-ARLYCK, Élisabeth (2001). « Articles en tous genres : le composite », in Société d'Étude de la Littérature Française du $\mathrm{xx}^{\mathrm{e}}$ siècle, L'éclatement des genres au $\mathrm{XX}^{e}$ siècle. Paris : Presses de la Sorbonne Nouvelle, pp. 305-317.

CÉSAIRE, Aimé (1939). Cahier d'un retour au pays natal. Paris : Présence Africaine (1983).

CHEVRIER, Marc (2012). « La Fatigue linguistique de la France », [consulté le 11 / 03/ 2015]

$<$ URL : http://agora-2.org/francophonie.nsf/Documents/Anglomanie--

La_fatigue_linguistique_de_la_France_par_Marc_Chevrier>

Deleuze, Gilles et GUATTARI, Félix (1980). Mille plateaux, Capitalisme et schizophrénie 2, Paris : Minuit (2013 pour la présente édition).

DERRIDA, Jacques (2003 [1986]). Parages. Paris : Galilée.

KRISTEVA, Julia et al (2007). Diversité et culture. Paris : Cedex, La Documentation française, Cultures France (Collection Penser L'Europe).

SOCIÉTÉ D'ÉTUDE DE LA LITTÉRATURE FRANÇAISE DU XXe SIÈCLE. L'éclatement des genres au XXe siècle (2001). Paris : Presses de la Sorbonne Nouvelle.

FINKIELKRAUT, Alain (1987). Une voix vient de l'autre rive. Paris : Gallimard.

GLISSANT, Édouard (1990). Poétique de la relation, (Poétique III), Paris : Gallimard.

GOBARD, Henri (1976). L’Aliénation linguistique. Paris : Flammarion.

HALL, T. Edward (1963). La proxémique sur l'Internet en 2013, [consulté le 18-05-2012]

<URL : http://www.futura-sciences.com/fr/news/t/recherche/d/laproxemique-limportance-delespace-personnel_10042/>

HOFSTADTER, Douglas ([1985] 2000). Gödel, Escher, Bach. Les brins d'une guirlande éternelle, traduit de l'américain par Robert French et Jacqueline Henry. Paris : Dunod, 2000.

IVANOV, V.N., \& al. (1974). « Thèses pour l'étude sémiotique des cultures », Sémiotique. № 81-84.

LE CLÉZIO, Jean-Marie Gustave (1963). Le Procès-verbal. Paris : Gallimard (2010).

LOTMAN, Youri (1999). La Sémiosphère. Limoges : Presses Universitaires de Limoges.

MAALOUF, Amin (1998). Les Identités meurtrières. Paris : Grasset et Fasquelle.

MORIN, Edgar (2002). « L'Identité humaine », Le Français dans le monde, n 322.

ORGANISATION INTERNATIONALE DE LA FRANCOPHONIE / 40 ans, (2010). La Langue française dans le monde. Paris : Nathan.

ORSENNA, Erik (2002). Madame Bâ. Paris: Fayard/Stock.

PARENT, Roger (2009). Résoudre des conflits de culture / Essai de sémiotique culturelle appliquée. Canad: Les Presses de l'Université Laval.

PRIGOGINE, Ilya, STENGERS, Isabelle (1984). Order Out of Chaos. New York: Bantam. 
RENARD, Raymond (2000). Une éthique pour la francophonie / Questions de politique linguistique. Paris : Didier Erudition.

ROSENSTIEHL, Pierre, PETITOT Jean (1974). « Automate asocial et système acentré », Communications, $\mathrm{n}^{\circ} 22,1974$, pp. 45-62.

SYN-THÈsES (2010). Revue annuelle. Traduction : médiation, négociation, communication. $\mathrm{N}^{\circ} 3$.

Département de Langue et de Littérature Française de l'Université Aristote de Thessalonique.

THIAM, Ndiassé (1997). « Alternance codique », in M. L. Moreau, Sociolinguistique : Les concepts de base. Hayen / Sprimont : P. Mardaga.

TISSIER, Rémy (2004). Le Dédale des disciples. Paris : L’Harmattan.

THIBAULT, Bruno \& MOSER, Keith (2012). J.-M. G. Le Clézio Dans la forêt des paradoxes. Paris :

L'Harmattan.

TOURAINE, Alain (1997). « Faux et vrais problèmes », in Une société fragmentée ? Le multiculturalisme en débat. Paris : La Découverte, pp. 291-319.

VAUGEOIS, Dominique (2001). «Conditions et fonctionnement de l'hétérogénéité générique dans Henri Matisse, Roman », in Société d'Étude de la Littérature Française du xx siècle. L'éclatement des genres au $\mathrm{XX}^{e}$ siècle. Paris : Presses de la Sorbonne Nouvelle, pp. 35-47.

VENTURA, Héliane, MOTTET, Philippe (2009). Pratiques de la transgression dans la littérature et les arts visuels. Actes du colloque international « Les transgressions verbi-voco-visuelles ». Québec : L 'instant même.

VLASSELAER, Jean-Jacques Van (1994). SGAV : paradigme ouvert sur l'apprentissage / Essais de didactique des langues. Paris : Didier Érudition, Mons, CIPA.

WATTZLAWICK, \& al (1972). Une logique de la communication. Paris : Seuil.

WESTPHAL, Bertrand (2007). La Géocritique. Paris : Minuit.

\section{NOTES}

1. Le pérégrinisme s'insère dans un texte comme un élément étranger, sans toujours être suivi d'une traduction ou d'une note métalinguistique.

2. Cette image renverrait aussi, par la légèreté qu'elle symbolise, aux opérations de l'imagination, dont la nature vagabonde et butineuse fait qu'elle passe le temps voletant de-ci de-là, sans méthode, à la quête d'une matière à fournir à l'artiste.

3. Lotman aborde la question du plurilinguisme sous l'angle de la sémiotique de la culture. La sémiotique étant à entendre comme » le milieu qui rend possible la création des signes ». À travers son étude, il valide la thèse de l'ambivalence des frontières. Selon lui, en raison de la porosité des frontières, les signes se transforment quand ils entrent en contact avec une autre culture.

4. Dans ce roman aux accents de programme esthétique, cet « écrivain de la rupture » accorde une certaine place à l'exploration du rôle du langage dans la société, aux différents avatars du langage, comme en témoignent les ruptures codiques notées ça et là dans le récit.

5. Nous avons paraphrasé Erik Orsenna qui a utilisé l'image du maillon et de la chaîne dans son Madame Bâ, Paris : Fayard/Stock, 2002, p. 323.

6. La proxémique : domaine qui étudie le rôle joué par l'espace physique dans la communication entre les personnes. Les bases de la proxémique ont été jetées en 1963 par l'anthropologue 
Edward T. Hall. À en croire celui-ci, il y a en général quatre « zones » de proximité :

- Intime (de $15 \mathrm{~cm}$ à $45 \mathrm{~cm}$ : pour embrasser, chuchoter).

- Personnel (de $45 \mathrm{~cm}$ à $1,2 \mathrm{~m}$ : pour les amis).

- Social (de $1,2 \mathrm{~m}$ à $3,6 \mathrm{~m}$ pour les connaissances).

Publique (plus de 3,6m). En ligne, http://www.futura-sciences.com/magazines/sciences/infos/ $\mathrm{actu} / \mathrm{d} /$ recherche-proxemique-importance-espace-personnel-10042/, visité ce 18-05-2012 à $21 \mathrm{~h} 1$. La communication entre la femme de Balingo et Sarah, nous la situons dans la "zone » dite intime puisque leurs fronts se sont touchés, la distance qui les sépare s'est réduite à presque néant comme l'indique les marqueurs spatiaux « près » et " proche ». Cela montre que, quand la corporalité a partie liée avec l'espace, elle n'empêche pas un dialogue authentique, mais permet de découvrir d'autres moyens de communication et de connaissance.

\section{RÉSUMÉS}

Estomper, voire abolir, les hiérarchies et faire chuter les enclos identitaires : voilà les nouveaux sillons qu'un des versants de l'esthétique moderne et contemporaine, représentée ici par Le Dédale des disciples de Rémy Tissier, se propose d'ouvrir. Le présent article se donne pour objet d'étudier les frictions par lesquelles s'opère la dissolution des hiérarchies et se constitue l'hétérogénéité sur la plate-forme linguistique qui donne ainsi lieu à une esthétique de l'ébranlement. Il se déploie suivant la programmation de deux escales : une première escale qui, analysant l'« hétérarchie » linguistique, montre que les langues, même les plus lilliputiennes, sont autant de clés ouvrant la réalité pour faire jaillir et saillir le différentiel. Chacune de ces langues est dépositaire et porteuse d'un empan de sens de l'expérience humaine. D'où la nécessité de maintenir la balance égale entre les langues pour le respect et le bien de leurs dignités respectives. Une seconde escale permettra de se pencher sur la dimension prévisionnelle du parti pris transgressif pour faire observer que l'autre enjeu du plurilinguisme résiderait dans sa capacité à tordre le cou à l'absolutisme et à faire tourner à plein régime la machine de la relativité pour un bien meilleur dessein et destin de l'humanité.

To blur, or even to abolish, hierarchies and to bring down the enclosures of identity - such are the new paths that one of the strains of modern and contemporary aesthetics, represented here by Rémy Tissier's Le Dédale des disciples, sets out to explore. The present article aims to study the frictions through which hierarchies are dissolved and heterogeneity is generated at the linguistic level, giving rise to an aesthetics of "disquiet". In a two-step argument, an analysis of linguistic "hierarchy "is first offered, showing that languages, even Lilliputian ones, are as many keys to approach reality, thereby enhancing the differences that matter. Every language is the guardian of a portion of the meaning of human experience. Hence the need to strike somewhat of a balance between languages, as a sign of respect for each one of them and to preserve their dignity. The second part of the study addresses the predictive dimension of the transgressive option, to conclude that the value of multilingualism is likely to reside in its ability both to shatter absolutism and to set the machinery of relativity to work at full speed, towards a better goal and a better destiny for all of mankind. 
INDEX

Keywords : language, equality, diversity, hierarchy, transgression

Mots-clés : langue, égalité, diversité, hiérarchie, transgression

\section{AUTEUR}

MAMADOU FAYE

Université de Cheikh Anta Diop/Dakar

ayserere[at\}yahoo.fr 